\title{
Erratum to: Responses of peripheral endocannabinoids and endocannabinoid-related compounds to hedonic eating in obesity
}

\author{
Alessio Maria Monteleone ${ }^{1} \cdot$ Vincenzo Di Marzo $^{2} \cdot$ Palmiero Monteleone $^{1,3}$. \\ Riccardo Dalle Grave ${ }^{4}$ Teresa Aveta ${ }^{2} \cdot$ Marwan El Ghoch $^{4} \cdot$ Fabiana Piscitelli $^{2}$. \\ Umberto Volpe $^{1} \cdot$ Simona Calugi ${ }^{4} \cdot$ Mario Maj $^{1}$
}

Published online: 2 May 2016

(C) Springer-Verlag Berlin Heidelberg 2016

\section{Erratum to: Eur J Nutr \\ DOI 10.1007/s00394-016-1153-9}

In the original publication of the article, the names of the authors Riccardo Dalle Grave and Marwan El Ghoch were incorrect in the original PubMed citation of this publication. The original article has also been updated accordingly.

The online version of the original article can be found under doi:10.1007/s00394-016-1153-9.

Vincenzo Di Marzo

vdimarzo@icb.cnr.it

$\bowtie$ Palmiero Monteleone

monteri@tin.it

1 Department of Psychiatry, University of Naples SUN, Naples, Italy

2 Endocannabinoid Research Group, Institute of Biomolecular Chemistry, Consiglio Nazionale delle Ricerche, 80078 Pozzuoli, NA, Italy

3 Neuroscience Section, Department of Medicine and Surgery, University of Salerno, 84081 Baronissi, Salerno, Italy

4 Department of Eating and Weight Disorders, Villa Garda Hospital, Garda, Verona, Italy 